\title{
Celastrol nanoparticles inhibit corneal neovascularization induced by suturing in rats
}

This article was published in the following Dove Press journal:

International Journal of Nanomedicine

2 March 2012

Number of times this article has been viewed

\section{Zhanrong $\mathrm{Li}^{\prime}$ \\ Lin Yao' \\ Jingguo $\mathrm{Li}^{2}$ \\ Wenxin Zhang' \\ Xianghua $\mathrm{Wu}^{\prime}$ \\ Yi Liu' \\ Miaoli Lin' \\ Wenru Su' \\ Yongping $\mathrm{Li}^{\prime}$ \\ Dan Liang'}

'State Key Laboratory of Ophthalmology, Zhongshan Ophthalmic Center, Sun Yat-Sen University, ${ }^{2}$ School of Chemistry and Chemical Engineering, Sun

Yat-Sen University, Guangzhou, People's Republic of China
Correspondence: Dan Liang State Key Laboratory of Ophthalmology, Zhongshan Ophthalmic Center, Sun Yat-Sen University, 54 Xianlie South Road, Guangzhou 510060 , People's Republic of China Tel +86 2087331766

Fax +86 208733 |93|

Email liangd2@mail.sysu.edu.cn
Purpose: Celastrol, a traditional Chinese medicine, is widely used in anti-inflammation and anti-angiogenesis research. However, the poor water solubility of celastrol restricts its further application. This paper aims to study the effect of celastrol nanoparticles (CNPs) on corneal neovascularization (CNV) and determine the possible mechanism.

Methods: To improve the hydrophilicity of celastrol, celastrol-loaded poly(ethylene glycol)block-poly( $\varepsilon$-caprolactone) nanopolymeric micelles were developed. The characterization of CNPs was measured by dynamic light scattering and transmission electron microscopy analysis. Celastrol loading content and release were assessed by ultraviolet-visible analysis and high performance liquid chromatography, respectively. In vitro, human umbilical vein endothelial cell proliferation and capillary-like tube formation were assayed. In vivo, suture-induced CNV was chosen to evaluate the effect of CNPs on CNV in rats. Immunohistochemistry for CD68 assessed the macrophage infiltration of the cornea on day 6 after surgery. Real-time quantitative reverse transcription-polymerase chain reaction and enzyme-linked immunosorbent assay were used to evaluate the messenger ribonucleic acid and protein levels, respectively, of vascular endothelial growth factor, matrix metalloproteinase 9 , and monocyte chemoattractant protein 1 in the cornea.

Results: The mean diameter of CNPs with spherical shape was $48 \mathrm{~nm}$. The celastrol loading content was $7.36 \%$. The release behavior of CNPs in buffered solution ( $\mathrm{pH} 7.4)$ showed a typical two-phase release profile. CNPs inhibited the proliferation of human umbilical vein endothelial cells in a dose-independent manner and suppressed the capillary structure formation. After treatment with CNPs, the length and area of CNV reduced from $1.16 \pm 0.18 \mathrm{~mm}$ to $0.49 \pm 0.12 \mathrm{~mm}$ and from $7.71 \pm 0.94 \mathrm{~mm}^{2}$ to $2.29 \pm 0.61 \mathrm{~mm}^{2}$, respectively. Macrophage infiltration decreased significantly in the CNP-treated corneas. CNPs reduced the expression of vascular endothelial growth factor, matrix metalloproteinase 9 , and monocyte chemoattractant protein 1 in the cornea on day 6 after suturing.

Conclusion: CNPs significantly inhibited suture-induced CNV by suppressing macrophage infiltration and the expression of vascular endothelial growth factor and matrix metalloproteinase 9 in the rat cornea.

Keywords: celastrol, PEG-b-PCL nanopolymeric micelles, corneal neovascularization, macrophages, VEGF, MMP-9

\section{Introduction}

Corneal neovascularization (CNV) is a leading aspect of the pathogenesis of many sight-threatening disorders, such as corneal graft rejection, corneal chemical injury, and infection. ${ }^{1,2}$ The process of new blood vessel formation requires proliferation, migration, and capillary tube formation by endothelial cells..$^{3,4}$ The infiltration of inflammatory 
cells and the release of several angiogenesis-related mediators contribute to multiple aspects of CNV. ${ }^{5,6} \mathrm{CNV}$ is also controlled by the interaction of endothelial cells with the extracellular matrix and the balance between proangiogenic and anti-angiogenic mediators. An imbalance toward proangiogenic factors, such as vascular endothelial growth factor (VEGF), tumor necrosis factor- $\alpha$ (TNF- $\alpha$ ), and matrix metalloproteinases (MMPs), can upregulate angiogenesis. ${ }^{7}$

Macrophages play major roles in many angiogenesis models by secreting several inflammatory and angiogenic mediators. ${ }^{8-10}$ Macrophage infiltration promotes sutureinduced CNV. ${ }^{11}$ The authors' previous unpublished studies showed that macrophages can induce CNV by upregulating VEGF and MMP-9.

Celastrol, also known as tripterine, is a traditional Chinese herb derived from Trypterygium wilfordii. ${ }^{12}$ Celastrol exhibits potent anti-angiogenic and anti-inflammatory activities by modulating multiple pro-angiogenic and pro-inflammatory cytokines, such as hypoxia-inducible factor- $1 \alpha$, TNF- $\alpha$, VEGF, and cyclooxygenase $2 .{ }^{13-15}$ These mediators play major roles in the proliferation of endothelial cells and the progression of angiogenesis. In macrophages, celastrol also inhibits lipopolysaccharide- and interferon- $\gamma$-induced production of TNF- $\alpha$, nitric oxide, interleukin- $1 \beta$ (IL-1 $\beta$ ), prostaglandin E2, and IL-6. ${ }^{16-19}$ Although celastrol has shown potent anti-inflammatory and anti-angiogenic activities in many models, it is unknown whether celastrol can modulate CNV and inflammation.
Despite the powerful beneficial bioactivity of celastrol, its clinical use has been limited mainly due to its poor water solubility. Therefore, it will be necessary to improve its water solubility while still retaining its activities in order to develop new celastrol-based therapeutics. Nanoformulated poly(ethylene glycol)-block-poly( $\varepsilon$-caprolactone) (PEG-b-PCL) micelles have shown promise in improving the hydrophilicity of drugs and enabling their extended release..$^{20,21}$

The aim of the present study was to determine the effects of celastrol-loaded PEG-b-PCL nanopolymeric micelles on corneal angiogenesis induced by suturing in the rat cornea and to determine the possible mechanism involved.

\section{Materials and methods Preparation of celastrol-loaded polymer micelles}

Chinese herbal celastrol (Figure 1A) was purchased from the Shanghai Institute of Materia Medica, Chinese Academy of Sciences (Shanghai, China). Celastrol-loaded PEG-bPCL micelles were prepared by dissolving PEG-b-PCL (2000:1000, weight-average molecular weight/number average molecular weight: 1.18; JCS Biopolytech Inc, Toronto, $\mathrm{ON}$, Canada; Figure 1B) (10 mg) and celastrol (2 mg) in chloroform $(2 \mathrm{~mL})$ and adding this solution dropwise to double-distilled water $(20 \mathrm{~mL})$ under ultrasonic agitation using a sonic dismembrator (model 60; Thermo Fisher Scientific, Pittsburgh, PA) at a power level of 30 . The organic solvent was then removed by vacuum distillation using a

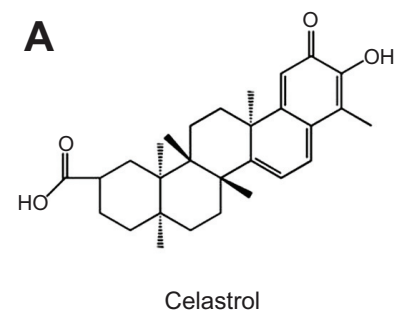

B
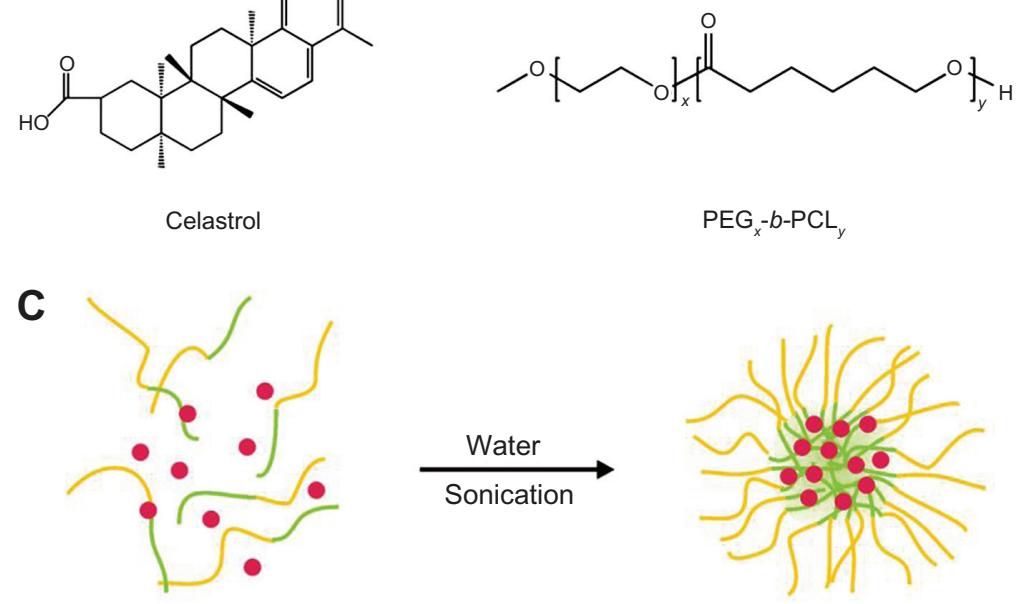

- Celastrol

PEG-b-PCL copolymer

Figure I The structure of (A) celastrol and (B) poly(ethylene glycol)-block-poly(E-caprolactone) nanopolymeric micelles and (C) schematic illustration of celastrol-loaded micelle formation.

Abbreviation: PEG-b-PCL, poly(ethylene glycol)-block-poly(e-caprolactone). 
rotary evaporator to allow micelle formation. The samples were further concentrated and washed three times to remove insoluble material and unincorporated drug through a $0.22 \mu \mathrm{m}$ polyethersulfone filter. A schematic illustration of celastrol-loaded micelle formation is shown in Figure 1C.

\section{Determination of nanopolymeric micelle diameters}

The particle size of nanopolymeric micelles was measured by dynamic light scattering. The suspension was examined at $25^{\circ} \mathrm{C}$ on 90Plus/BI-MAS equipment (Brookhaven Instruments Corporation, Holtsville, NY). The related data were collected by an autocorrelator with a detection angle of scattered light of $90^{\circ}$.

\section{Transmission electron microscopy (TEM) analysis}

The celastrol-loaded nanopolymeric micelles were characterized using TEM. Samples for TEM analysis were prepared on copper grids coated with amorphous carbon. A small drop of uranyl acetate solution ( $2 \%$ weight/volume in water) was added to the copper grid for negative staining of samples, and the grid was blotted with filter paper after 1 minute. TEM measurements were performed on an H-7650 TEM (Hitachi High Technologies, Tokyo, Japan) operated at $80 \mathrm{kV}$.

\section{Evaluation of celastrol loading content}

The celastrol loading content, defined as the weight percentage of celastrol in micelle, was quantified by ultraviolet-visible analysis using an ultraviolet-visible spectrophotometer (UV-2000; Unico Instruments Co, Ltd, Shanghai, China). First, celastrol-loaded micelle solutions were frozen and lyophilized to yield the solid micelle samples. And then the dried micelle samples were weighed and redissolved in a mixture of dimethyl sulfoxide and chloroform (1:1, volume/volume) for the ultraviolet-visible measurement. Celastrol solutions of various concentrations were prepared, and the absorbance at $425 \mathrm{~nm}$ was measured to generate a calibration curve for the celastrol loading content calculations from micelles.

\section{In vitro release of celastrol from polymer micelles}

Freeze-dried micelle samples (12 mg each) were resuspended in $3 \mathrm{~mL}$ phosphate buffered saline $(\mathrm{pH} \mathrm{7.4)}$ and then transferred into a dialysis bag (molecular weight cut-off: $14,000 \mathrm{Da}$ ). The bag was placed into the same buffered solution $(50 \mathrm{~mL})$. Release study was performed at $37^{\circ} \mathrm{C}$ in an incubator shaker $(\mathrm{C} 24$; New Brunswick
Scientific, Enfield, CT). At selected time intervals, buffered solution outside the dialysis bag was removed for reversephase high performance liquid chromatography (Dionex UltiMate $^{\circledR}$; Thermo Fisher; $5 \mu \mathrm{m}, 4.6 \times 250 \mathrm{~mm}$ columns) analysis and replaced with fresh buffer solution. Celastrol concentration was calculated based on the absorbance intensity at $425 \mathrm{~nm}$. In the assessment of drug release behavior, the cumulative amount of released drug was calculated and the percentage of drug released from micelles was plotted against time.

\section{Cell culture}

The human umbilical vein endothelial cells (HUVECs) (EA. hy 926; American Type Culture Collection, Manassas, VA) were cultured as described previously. ${ }^{22}$ Briefly, cells were grown in Gibco ${ }^{\circledR}$ Dulbecco's modified Eagle's medium (with 4.5 g/mL glucose; Invitrogen Life Technologies, Carlsbad, CA) supplemented with Gibco $^{\circledR} 10 \%$ fetal bovine serum (Invitrogen) at $37^{\circ} \mathrm{C}$ in a $5 \%$ carbon dioxide atmosphere.

\section{In vitro HUVEC proliferation assays}

Inhibitory effects of celastrol particles on HUVEC proliferation were measured using a water-soluble tetrazolium salt (WST) reagent (2-[2-methoxy-4-nitrophenyl]3-[4-nitrophenyl]-5-[2,4-disulfophenyl]-2H, WST-8; Nacalai Tesque Inc, Kyoto, Japan) according to the manufacturer's protocol. EA.hy 926 cells were plated at a density of $2 \times 10^{3}$ per well in triplicate in fresh medium. After 24 hours, cells were respectively treated with medium containing different concentrations of celastrol nanoparticles (CNPs) $(0,1.7$, $3.4,6.8,13.6$, or $27.2 \mu \mathrm{g} / \mathrm{mL}$; celastrol loading content: $7.36 \%$ ) and the same dosage of PEG-b-PCL micelles without celastrol, and then cultured for an additional 72 hours. This treatment was followed by the addition of $10 \mu \mathrm{L}$ of WST solution to each well, and the cells were incubated for 4 hours at $37^{\circ} \mathrm{C}$. The absorbance was measured at $450 \mathrm{~nm}$. Changes in cell morphology were captured after 72 hours of treatment with CNPs. The antiproliferation effect of free celastrol dissolved in dimethyl sulfoxide ( $0.2 \%$ volume/volume $)$ - on HUVECs $(0,0.125,0.25,0.5,1$, or $2 \mu \mathrm{g} / \mathrm{mL}$ concentration) was also assessed to compare with the effect of CNPs. The measurement protocol was as described above.

\section{Capillary-like tube formation assay}

Tube formation was evaluated as described previously. ${ }^{23,24}$ Briefly, the stock solution of Matrigel ${ }^{\mathrm{TM}}$ (BD Biosciences, San Jose, CA) was diluted 1:3 with Dulbecco's modified Eagle's medium, and $50 \mu \mathrm{L}$ of diluted Matrigel solution 
was then added into each well of an ice-cold 96-well plate. The plates were incubated at $37^{\circ} \mathrm{C}$ for 30 minutes to convert this into a gelatinous layer. EA.hy 926 cells were pretreated with a dilution of CNPs $(13.6 \mu \mathrm{g} / \mathrm{mL}$ or $27.2 \mu \mathrm{g} /$ $\mathrm{mL}$; celastrol loading content: $7.36 \%$ ) for 30 minutes and then seeded onto the Matrigel layers in the 96-well plate at a density of $5 \times 10^{4}$ cells/well. After 10 hours, the tubular structure of endothelial cells was observed under an inverted microscope (magnification: $\times 200$; Olympus Corporation, Tokyo, Japan). Three independent experiments were performed.

\section{Animal treatment}

To clarify the effects of CNPs on CNV, the modified model of suture-induced CNV was used, as described previously. ${ }^{25,26}$ Before surgery, all female Sprague-Dawley rats (weighing 200-220 g) were deeply anesthetized. CNV was induced by suturing (two stitches) the temporal cornea with 10-0 nylon (Alcon Laboratories, Inc, Fort Worth, TX). The distance between the stitches and the limbus was approximately $1.5 \mathrm{~mm}$. There was a distance of $1 \mathrm{~mm}$ between the two stitches. Tobramycin ointment was applied to the cornea to prevent corneal inflammation. To examine the anti-angiogenic activity of celastrol, $0.1 \mathrm{~mL}$ CNPs were administrated by subconjunctival injection at a concentration of $5.44 \mathrm{mg} / \mathrm{mL}$ (celastrol loading content: $7.36 \%$ ) for each rat on day 0 and day 3 after surgery. The control group was exposed to the same dosage of blank PEG-bPCL micelles without celastrol. CNV was observed by slit-lamp microscopy (SL120; Carl Zeiss AG, Oberkochen, Germany) every day until day 6 . Eye irritation was recorded according to the Draize eye test. ${ }^{27}$ The doses and time points used were based on the results of the authors' unpublished observations.

\section{Quantification of CNV}

CNV was quantified according to a previous report. ${ }^{28}$ Briefly, on the sixth postoperative day, the rats were anesthetized again with sodium pentobarbital $(50 \mathrm{mg} / \mathrm{kg}$ body weight, intraperitoneally) and administered heparin at a dose of 2000 units $/ \mathrm{kg}$ body weight via tail vein injection to prevent blood clotting. Thirty minutes after infusion, all rats were perfused with $10 \mathrm{~mL}$ of Higgins waterproof India ink (Sanford, Oak Brook, IL) through the thoracic aorta to visualize corneal vessels. The eyes were enucleated and fixed in 10\% neutralized buffered formaldehyde for 24 hours, and then the corneas were dissected and mounted on slides.
Corneal images were captured and analyzed with a slitlamp microscope (Carl Zeiss SL120) by an investigator who was blinded to the treatment assignment. The length and area of CNV were measured with Image-Pro ${ }^{\circledR}$ Plus 5.1 (Media Cybernetics Inc, Bethesda, MD), according to a previous report. $^{28}$

\section{Histology and immunohistochemistry}

Six days after surgery, the eyes were enucleated, fixed in $10 \%$ neutralized buffered formaldehyde and embedded in paraffin. Thereafter, the tissue was cut into $5-\mu \mathrm{m}$ thick sections and stained with hematoxylin and eosin. To identify infiltrating macrophages, immunohistochemistry for CD68 (Santa Cruz Biotechnology, Santa Cruz, CA) was carried out on deparaffinized sections. Corneal morphology and images of the infiltrated macrophages were assessed by light microscopy. The number of infiltrated macrophage (CD68+) cells in the corneal stroma was counted in five randomly selected fields $(\times 400)$ of the immunofluorescence-stained slides by an experienced and independent cell scientist who was blinded to the treatment assignment.

\section{Real-time quantitative reverse transcription-polymerase chain reaction (PCR)}

Corneas were harvested on day 6 after surgery. Total ribonucleic acid (RNA) was isolated with TRIzol ${ }^{\circledR}$ reagent (Invitrogen), according to the manufacturer's instructions. It was extracted with chloroform and proazamine, and transcribed into complementary DNA using the Superscript ${ }^{\mathrm{TM}}$ First-strand Synthesis System for Reverse Transcription-PCR (Invitrogen), according to the manufacturer's instructions. Quantitative real-time PCR was carried out using a 7100 RealTime PCR System (Applied Biosystems, Foster City, CA) with SYBR-Green 1 (Takara Bio Inc, Otsu, Japan) and analyzed with the ABI PRISM ${ }^{\circledR} 7000$ Sequence Detection System (Applied Biosystems). The primers used were as follows: VEGF, 5'-GTCCTCACTTGGATCCCGACA-3' (forward) and $5^{\prime}$-CCTGGCAGGCAAACAGACTTC-3' (reverse); MMP-9: 5'-AGCCGGGAACGTATCTGGA-3' (forward) and 5'-TGGAAACTCACACGCCAGAAG-3' (reverse); monocyte chemoattractant protein 1 (MCP-1): 5'-CTATGCAGGTCTCTGTCACGCTTC-3' (forward) and 5'-CAGCCGACTCATTGGGATCA-3' (reverse); and $\beta$-actin: $5^{\prime}$-GGAGATTACTGCCCTGGCTCCTA-3' (forward) and 5'-GACTCATCGTACTCCTGCTTGCTG-3' (reverse). All expression levels were normalized to $\beta$-actin in the normal corneas. 


\section{Enzyme-linked immunosorbent assay (ELISA)}

VEGF, MMP-9, and MCP-1 concentrations in the cornea were determined using specific ELISA kits (R\&D Systems Inc, Minneapolis, MN), according to the manufacturer's instructions. On day 6 after surgery, the corneal tissues were collected in two groups. The proteins were extracted and stored at $-80^{\circ} \mathrm{C}$ until assaying for VEGF, MMP-9, and MCP-1.

\section{Statistical analysis}

Statistical analysis was performed using IBM SPSS software for Windows (v 16.0; SPSS Inc, Chicago, IL). All data are presented as mean \pm standard deviation. Differences between experimental and control groups were analyzed by independent-sample Student's $t$-test. $P$ values $<0.05$ were considered statistically significant.

\section{Results}

The aim of this study was to investigate whether CNPs can suppress corneal angiogenesis and, if so, through what mechanism. To answer these questions, several in vitro and in vivo assays were used, including an analysis of corneal angiogenesis in vivo and an analysis of cell proliferation, cytotoxicity, and cellular morphology of HUVECs in vitro.

\section{Characterization of celastrol-loaded nanopolymeric micelles and celastrol loading content}

Celastrol-loaded PEG-b-PCL micelles were characterized before analyzing the anti-angiogenic effect of celastrol. The size and shape of the celastrol-loaded PEG-b-PCL micelles were determined by dynamic light scattering and TEM, respectively. From the dynamic light scattering data presented in Figure 2A, the mean diameter of blank and celastrol-loaded nanopolymeric micelles was $36 \mathrm{~nm}$ and $48 \mathrm{~nm}$, respectively. The electron photomicrograph of the nanopolymeric micelles shown in Figure 2B illustrates their spherical shape. TEM also confirmed their diameter at approximately $48 \mathrm{~nm}$. The celastrol loading content of PEG-b-PCL micelle was 7.36\%.

\section{In vitro celastrol release study}

The in vitro release behavior of celastrol-loaded micelles in buffered solutions ( $\mathrm{pH}$ 7.4) was studied. As shown in Figure 3, a typical two-phase release profile was observed. That is, a relatively rapid release in the first stage followed by a sustained and slow release over a prolonged time of up to several weeks.

\section{CNPs inhibit the growth and capillary structure formation of HUVECs}

The effect of CNPs on the growth viability of HUVECs was investigated using WST assays. The morphologic changes of cells showed that increasing the amount of CNPs decreased the number of HUVECs (Figure 4A). CNPs reduced the viability of EA.hy 926 cells in a dose-dependent manner. At $6.8 \mu \mathrm{g} / \mathrm{mL}, 13.6 \mu \mathrm{g} / \mathrm{mL}$, and $27.2 \mu \mathrm{g} / \mathrm{mL}$ CNP concentration, the viability of EA.hy 926 cells was reduced significantly (Figure 4B). However, the blank micelles showed no obvious toxicity on HUVECs in the tested concentration range and time (Figure 4B). The half maximal inhibitory concentration $\left(\mathrm{IC}_{50}\right)$ value of the antiproliferation effect of CNPs at 72 hours was $6.875 \mu \mathrm{g} / \mathrm{mL}$ (celastrol loading
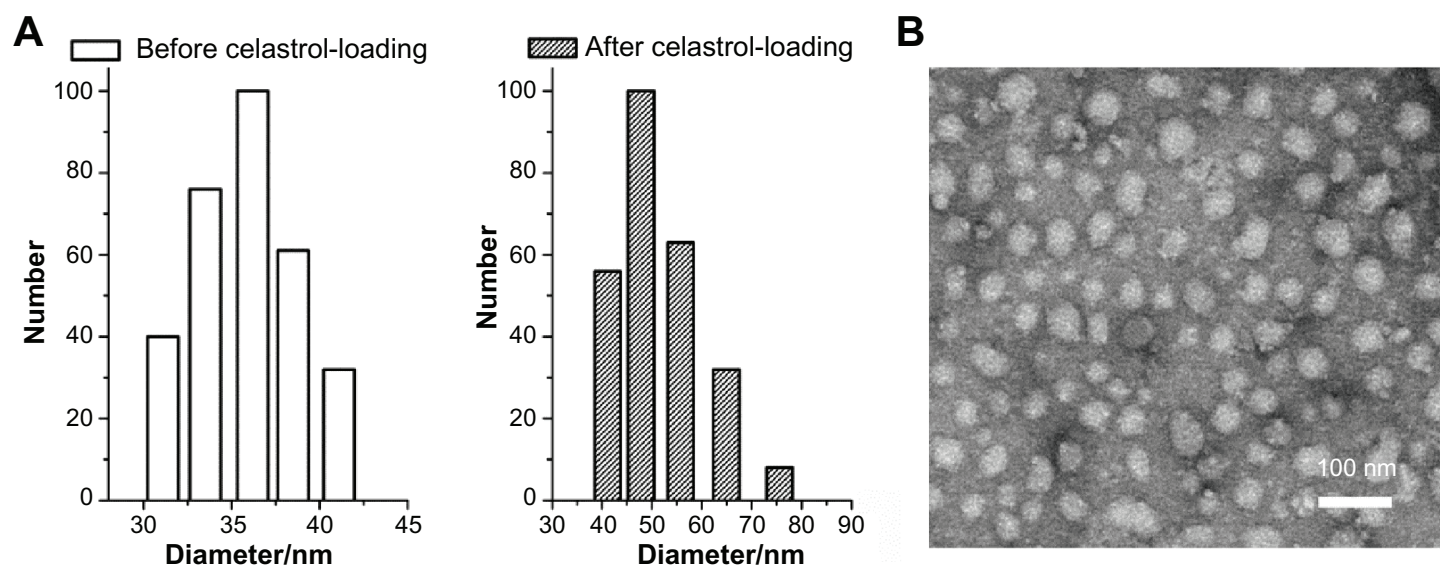

Figure 2 Average diameter of micelles before and after celastrol loading and transmission electron microscopy images of celastrol-loaded micelles. The particle size of nanopolymeric micelles was measured by dynamic light scattering. (A) Dynamic light scattering histogram reveals the size distribution of $48 \mathrm{~nm}$ celastrol polymeric micelles based on a poly(ethylene glycol)-block-poly( $\varepsilon$-caprolactone) copolymer (mean diameter: $36 \mathrm{~nm}$ ). The celastrol nanoparticles were characterized using transmission electron microscopy. (B) Transmission electron microscopy image of the micelle sample after negative staining with $2 \%$ uranyl acetate solution shows their spherical shape. 


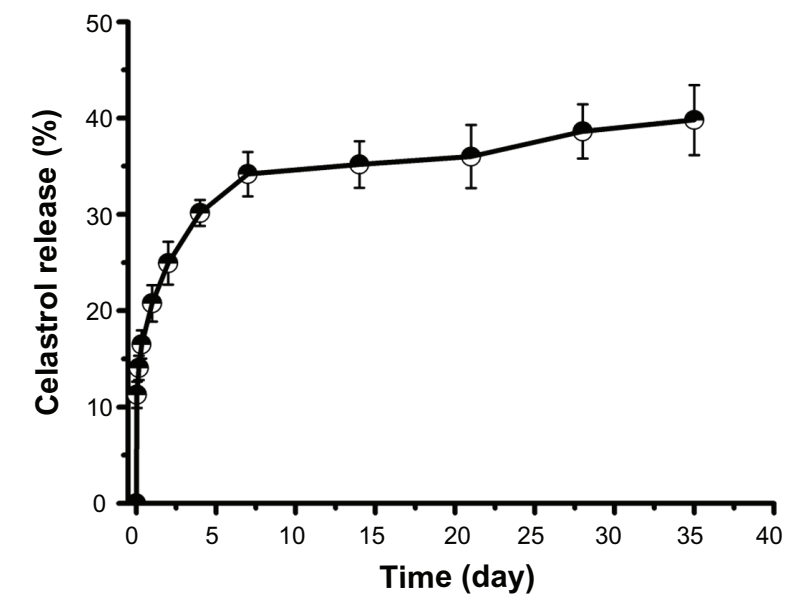

Figure 3 In vitro drug release profiles of celastrol from poly(ethylene glycol)block-poly( $\varepsilon$-caprolactone) micelles (celastrol loading content: $7.36 \%)$ in phosphate buffered saline $\left(\mathrm{pH} \mathrm{7.4)}\right.$ at $37^{\circ} \mathrm{C}$. A typical two-phase release profile contained a burst release in the first stage followed by a sustained and slow release over a prolonged time of up to several weeks.

Note: The data are presented as mean \pm standard deviation $(n=3)$.

content: $7.36 \%$ ). And the $\mathrm{IC}_{50}$ value of free celastrol on EA.hy 926 cells was $0.325 \mu \mathrm{g} / \mathrm{mL}$.

To further characterize the effect of celastrol on angiogenesis, an analysis of how celastrol regulates capillary tubule formation in HUVECs was done. When EA.hy 926 cells were seeded on Matrigel, tubular-like structures formed. However, treatment with $13.6 \mu \mathrm{g} / \mathrm{mL}$ or $27.2 \mu \mathrm{g} / \mathrm{mL}$ (celastrol loading content: $7.36 \%$ ) of CNPs abolished the tubule formation (Figure 4C).

\section{CNPs suppress corneal angiogenesis in a rat model}

To investigate the effect of CNPs on CNV in vivo, $0.1 \mathrm{~mL}$ of CNPs by subconjunctival injection was administered at a concentration of $5.44 \mathrm{mg} / \mathrm{mL}$ on day 0 and day 3 after suture surgery in a rat model. New blood vessel buds appeared and began to develop into the corneal suture on day 1 or 2 after surgery. Abundant CNV reached its peak on day 6 and began to regress after day 8 in the control group. Corneal edema was observed during neovascularization (Figure 5A). However, shorter and fewer new corneal vessels and substantially less edema were found in the celastrol-treated group (Figure 5B). Corneal angiogenesis produced a black color after ink perfusion on day 6 in both groups (Figure $5 \mathrm{C}$ and D). Administration of $0.544 \mathrm{mg}$ of CNPs reduced the length and area of CNV. The average length and area were $1.16 \pm 0.18 \mathrm{~mm}$ and $7.71 \pm 0.94 \mathrm{~mm}^{2}$, respectively, in the control group on day 6 after surgery, whereas the average length and area in the celastrol group decreased to $0.49 \pm 0.12 \mathrm{~mm}$ and $2.29 \pm 0.61 \mathrm{~mm}^{2}$, respectively (both $P$ values $<0.001$ )
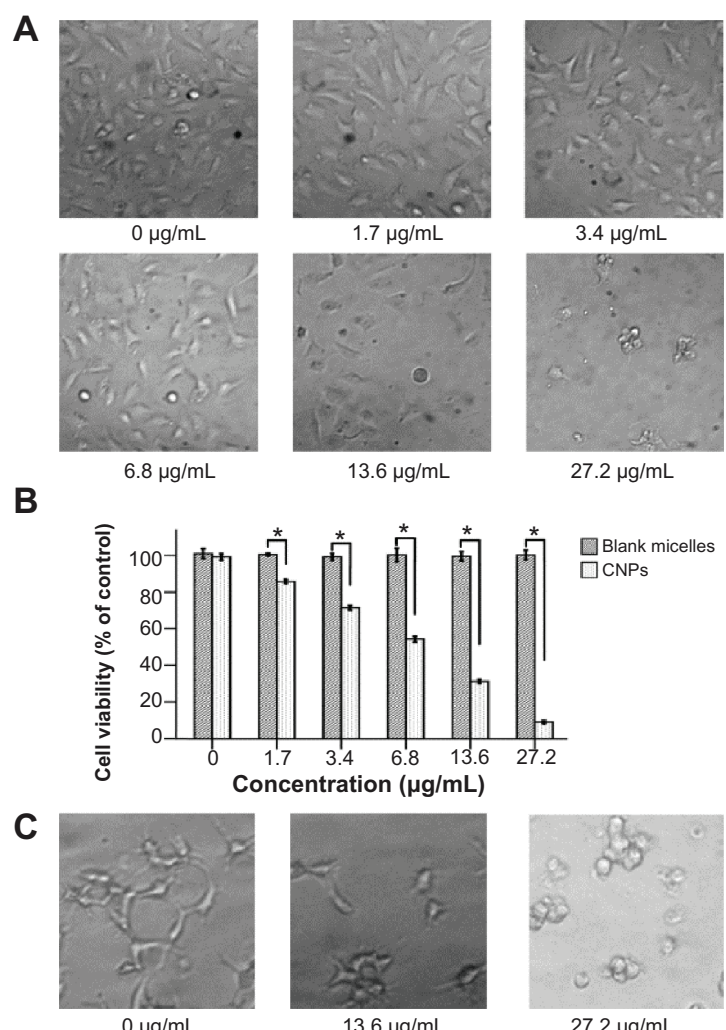

Figure 4 Celastrol nanoparticles dose-dependently inhibit the growth and capillary structure formation of human umbilical vein endothelial cells (EA.hy 926). (A) Morphological variations of EA.hy 926 cells treated with celastrol nanoparticles $(0,1.7,3.4,6.8,13.6$, or $27.2 \mu \mathrm{g} / \mathrm{mL})$ for 72 hours. (B) EA.hy 926 cells treated with either celastrol nanoparticles or blank micelles $(0,1.7,3.4,6.8,13.6$, or $27.2 \mu \mathrm{g} / \mathrm{mL}$ ) for 72 hours. Water-soluble tetrazolium salt assay was used to determine cell viability after exposure to the nanoparticles. The blank micelles without celastrol showed a cell viability of above $95 \%$. Increasing the amount of celastrol nanoparticles increased the inhibition effect on the EA.hy 926 cells. (C) Celastrol nanoparticles inhibited the tube formation of endothelial cells in Matrigel ${ }^{\mathrm{TM}}$ (BD Biosciences, San Jose, CA). EA.hy 926 cells pretreated with or without celastrol nanoparticles were placed in a 96-well plate coated with Matrigel $\left(5 \times 10^{4}\right.$ cells per well). After 10 hours, the tubular structure of the endothelial cells was photographed (magnification: $\times 200$ ).

Notes: The data are presented as mean \pm standard deviation for three independent experiments performed in triplicate. Statistical significance was based on the difference when compared with the control; $* P<0.0$ I.

(Figure 5E and F). No eye irritation, corneal epithelial defect, or other adverse complications related to the topical CNPs were observed in any of the tested animals.

\section{Celastrol nanopolymeric micelles inhibit neovascularization and macrophage infiltration in the rat cornea}

Histologic analysis of the cornea revealed that CNP treatment led to a significant reduction in the number of infiltrating inflammatory cells in the treated group when compared with the control group. New blood vessels were inhibited and corneal edema was also distinctly mitigated by celastrol (Figure 6A and B). The number of infiltrating macrophages was significantly reduced in the cornea on day 6 after 

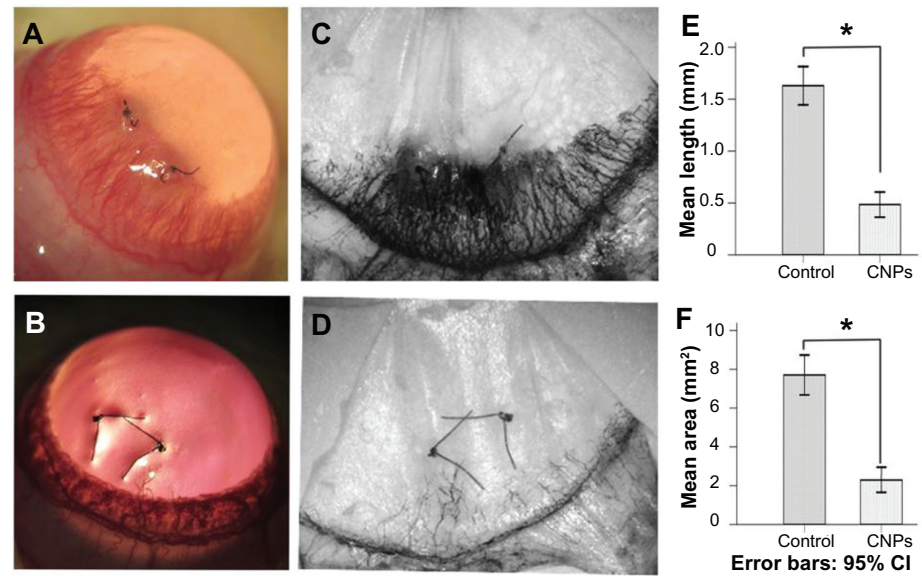

Figure 5 Inhibitory effects of celastrol nanoparticles on suture-induced corneal neovascularization in rats. Animals were sutured (two stitches) in the temporal cornea, and then treated with subconjunctival injection of celastrol nanoparticles ( $0.544 \mathrm{mg}$ per rat) on day 0 and day 3 after surgery. Slit-lamp microphotographs of corneas were taken after mydriasis on day 6 after surgery. The pictures show $(\mathbf{A})$ an intensive corneal neovascularization in response to suturing and (B) a marked inhibition of neovascularization by celastrol nanoparticles. The images were taken by perfusing the cornea with India ink on day 6 after suturing. Note the distinct suppression of corneal neovascularization by celastrol nanoparticles. (C) Abundant corneal neovascularization reached the sutures. (D) Shorter and fewer areas of corneal neovascularization were found in the celastroltreated group. (E and $\mathbf{F}$ ) show the inhibition effect of celastrol nanoparticles on the length and area of corneal neovascularization, respectively.

Notes: The length and area of corneal neovascularization were measured with Image-Pro ${ }^{\circledR}$ Plus $5 . I$ (Media Cybernetics Inc, Bethesda, MD) in triplicate for each cornea. The data are presented as mean \pm standard deviation of five rats. $* P<0.00$ I versus the control group (magnification: $\times 25)$.

Abbreviations: $\mathrm{Cl}$, confidence interval; CNPs, celastrol nanoparticles.

surgery in the celastrol group compared to the control group $(P<0.001$; Figure 6C-E). Histopathological evaluation was performed three times for six sections of each group.

\section{CNPs decrease angiogenesis-related factor messenger RNA (mRNA) levels in the cornea}

To further study the effect of CNPs on CNV, quantitative real-time reverse transcription-PCR was performed to determine the relative mRNA expression of VEGF, MMP-9, and MCP-1 on day 6 after surgery in rat corneas. Normal corneas were used as the control. The mRNA expression of the normal corneas was arbitrarily set as 1.0. As shown in Figure 7, CNPs significantly decreased the mRNA expression of VEGF, MMP-9, and MCP-1 in the cornea $(P<0.001)$. When compared with the control, a significant decrease in the expressions of VEGF (5.89-fold versus 1.69-fold) and MCP-1 (10.11-fold versus 3.90-fold) and a moderate decrease
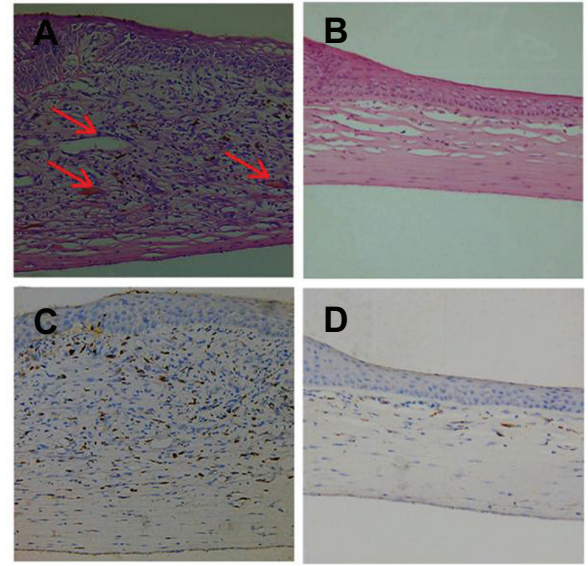

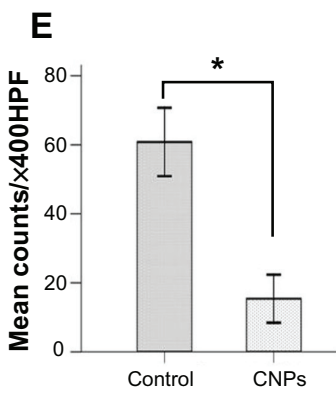

Error bars: $95 \% \mathrm{Cl}$

Figure 6 Celastrol nanoparticles inhibit neovascularization and macrophage infiltration in rat cornea. Rats were treated with celastrol nanoparticles ( 0.544 mg per rat) on day 0 and day 3 after surgery. Corneal tissue was fixed in $10 \%$ neutralized buffered formaldehyde, and $5 \mu$ m paraffin sections were stained with hematoxylin and eosin. (A) Celastrol nanoparticle-treated group showed significantly inhibited neovascularization and inflammatory cell infiltration in cornea on day 6 after surgery when compared with (B) the control group. Red arrows show new blood vessels. Corneal sections were processed by anti-rat CD68 staining in (C) celastrol nanoparticle-treated group and (D) the control group. Celastrol nanoparticles suppressed macrophage infiltration in the cornea on day 6 after surgery. The number of infiltrated macrophage (CD68+) cells in the corneal stroma was counted in five randomly selected fields $(\times 400)$ of the immunofluorescence-stained slides.

Notes: The number of infiltrating macrophages was analyzed by IBM SPSS ( $v$ I6.0; SPSS Inc, Chicago, IL). The data are presented as mean \pm standard deviation (magnification: $\times 100$ ). Statistical significance is based on the difference when compared with the control; $* P<0.00 \mathrm{I}$.

Abbreviation: $\mathrm{Cl}$, confidence interval. 

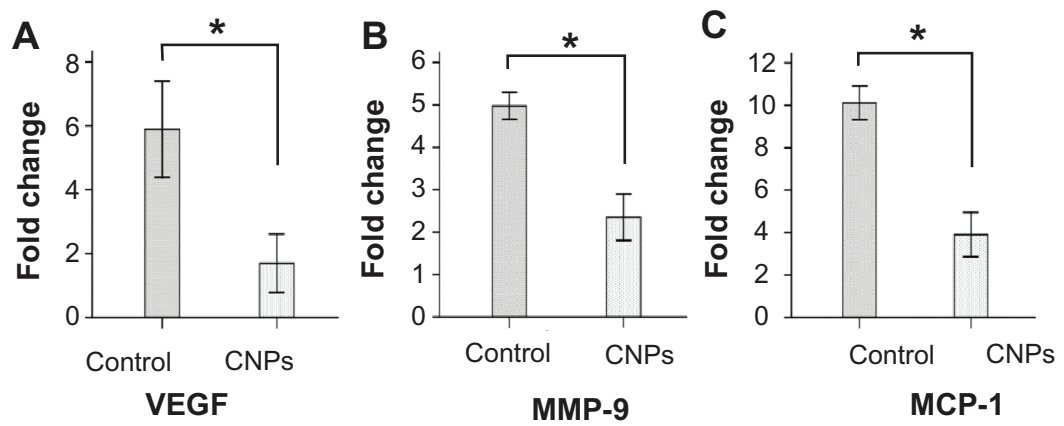

Figure 7 Celastrol nanoparticles inhibit the expression of angiogenic genes. Corneas were harvested on day 6 after surgery, followed by messenger ribonucleic acid expression analysis using real-time reverse transcription-polymerase chain reaction. Normal corneas were used as the control. Messenger ribonucleic acid expression of vascular endothelial growth factor, matrix metalloproteinase 9, and monocyte chemoattractant protein I in the normal corneas were arbitrarily set as I.0. The $y$-axis shows the mean of the relative messenger ribonucleic acid expression of a certain gene compared to the normal cornea gene. Celastrol nanoparticles decreased the expression of (A) vascular endothelial growth factor, (B) matrix metalloproteinase 9, and (C) monocyte chemoattractant protein I messenger ribonucleic acid in the cornea on day 6 after surgery.

Notes: The data are presented as mean \pm standard deviation. Statistical significance is based on the difference when compared with the control; $* P<0.00 I$.

Abbreviations: CNPs, celastrol nanoparticles; MCP-I, monocyte chemoattractant protein I; MMP-9, matrix metalloproteinase 9; VEGF, vascular endothelial growth factor.

in MMP-9 (4.97-fold versus 2.35-fold) were observed in the CNP treatment group.

\section{CNPs inhibit production of angiogenesis-related cytokines}

The effects of CNPs on angiogenesis-related cytokines in the rat cornea were evaluated. The protein levels of VEGF, MMP-9, and MCP-1, as determined by ELISA were $125.04 \pm 20.68 \mathrm{pg} / \mathrm{mL}, 15.37 \pm 4.17 \mathrm{pg} / \mathrm{mL}$, and $3.81 \pm 0.23 \mathrm{ng} / \mathrm{mL}$, respectively, in the celastrol group and $3354.71 \pm 98.17 \mathrm{pg} / \mathrm{mL}, 140.82 \pm 8.06 \mathrm{pg} / \mathrm{mL}$, and $8.89 \pm 0.25 \mathrm{ng} / \mathrm{mL}$, respectively, in the control group on day 6 after surgery. As assayed by ELISA, VEGF, MMP-9, and MCP-1 production was significantly reduced by CNPs on day 6 after surgery $(P<0.001$ for each; Figure 8$)$.

\section{Discussion}

$\mathrm{CNV}$ is closely related to local inflammatory cell infiltration, and several chemokines and cytokines are involved in this process. ${ }^{29}$ Macrophages promote corneal angiogenesis by releasing several pro-angiogenic growth factors and matrix remodeling proteases. Because of the antiinflammatory and anti-angiogenic activities of celastrol, it was speculated that celastrol mediates corneal angiogenesis by modulating macrophage activity. However, the poor water solubility of celastrol restricted its application in vivo. In the present study, PEG-b-PCL nanopolymeric micelles were used as a celastrol delivery carrier and the effect of CNPs on the properties of HUVECs and sutureinduced $\mathrm{CNV}$ in rats was examined. The results indicate that CNPs significantly inhibited the proliferation and capillary structure formation of HUVECs and suppressed in vivo CNV by inhibiting macrophage infiltration, which in turn was accomplished by the downregulation of VEGF and MMP-9 in rat cornea.

PCL, as a hydrophobic polymer, is an ideal material for drug delivery and other applications. ${ }^{30,31}$ However, its low biocompatibility with soft tissue and rather slow
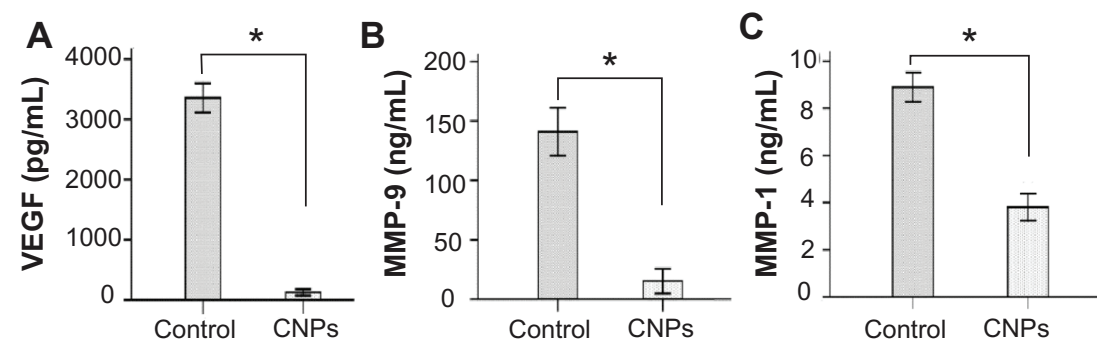

Figure 8 Celastrol nanoparticles inhibit the expression of angiogenic factor proteins in the cornea. Corneas were harvested on day 6 after surgery and the concentration of (A) vascular endothelial growth factor, (B) matrix metalloproteinase 9, and (C) monocyte chemoattractant protein I in the corneal tissue lysates were assessed by enzymelinked immunosorbent assay.

Notes: The data are presented as mean \pm standard deviation of three independent experiments. Statistical significance is based on the difference when compared with the control; $* P<0.001$.

Abbreviations: CNPs, celastrol nanoparticles; MCP-I, monocyte chemoattractant protein I; MMP-9, matrix metalloproteinase 9;VEGF, vascular endothelial growth factor. 
degradation restricts its further application. PEG - which is hydrophilic, nontoxic, and nonimmunogenic - can be attached to PCL to form PEG-b-PCL copolymers, ${ }^{32,33}$ which exhibit higher hydrophilicity and degradability, a more extended release, and better biocompatibility compared with the PCL homopolymer. ${ }^{34}$ Nanoformulated PEG-bPCL micelles as nanodrug delivery carriers have been used widely to improve the solubility of hydrophobic drugs..$^{21,34,35}$ The release behavior of PEG-b-PCL micelles has been studied extensively in vitro. ${ }^{36,37} \mathrm{In}$ the present study, PEGb-PCL micelle drug release showed a typical two-phase release profile consisting of a relatively rapid release in the first stage followed by a slow and sustained release, which is in accordance with a previous report. ${ }^{37}$ PEG-b-PCL is also a safe candidate for a sustained ophthalmic drug delivery system, based on toxicity evaluations. ${ }^{38}$ Therefore, PEG-b-PCL nanopolymeric micelles were chosen as the celastrol delivery carrier to further study the effect of CNPs in preventing CNV in rats. In the present study, an absence of eye irritation, corneal epithelial defects, and other adverse complications with the use of the topical CNPs was observed.

The suture-induced CNV model has been extensively used to understand the mechanism of angiogenesis and to evaluate the anti-angiogenic activities of many drugs. ${ }^{25,26}$ Some investigators have induced CNV by putting one stitch in the center of the cornea in rats, rabbits, or mice., ${ }^{4,25}$ Others have used more stitches and put them near the corneal limbus to induce more $\mathrm{CNV}^{26,40}$ According to the authors' unpublished observations, two stitches induced more CNV than one stitch placed the same distance from the corneal limbus. This finding supports those from previous reports. The distance of the two stitches from the limbus and the distance between the two stitches in the present study were in accordance with those of the corneal graft rejection model. This model probably best mimics angiogenesis in corneal graft rejections. In the present study, the two-stitch-induced CNV model was highly stable and reproducible, which makes it suitable for quantitative studies of CNV. It was also found that the effect of CNPs on CNV was superior with two subconjunctival administrations after surgery (day 0 and day 3) compared with only one administration at the same celastrol concentration. Therefore, two time points of subconjunctival injection were chosen, day 0 and day 3 after suturing. The CNPs significantly inhibited CNV compared with the control group.

Celastrol, a quinone methide triterpenoid from the "Thunder of God Vine," has various biological activities, including anti-angiogenesis, anti-inflammation, antitumor, and antioxidant. ${ }^{19,23,41}$ However, the process of angiogenesis is characterized by increased proliferation and the formation of capillary tubule-like structures by microvessel endothelial cells. ${ }^{3,4}$ In the present study, a dose-dependent inhibition of CNPs on HUVEC proliferation and tube formation was observed by WST- 8 assays with an $\mathrm{IC}_{50}$ value of $6.875 \mu \mathrm{g} / \mathrm{mL}$ (celastrol loading content: $7.36 \%$, concentration of celastrol: $0.506 \mu \mathrm{g} / \mathrm{mL}$ ) on EA.hy 926 cells. The $\mathrm{IC}_{50}$ value for free celastrol was $0.325 \mu \mathrm{g} / \mathrm{mL}$. The lower potency of micelle-delivered celastrol can be due to a time-consuming celastrol release from micelles, which is consistent with in vitro celastrol release studies. The inhibition effect of CNPs on HUVEC viability had a major role in its anti-angiogenesis activity by reducing new blood vessel development. This is consistent with the previously observed anti-angiogenic effect of celastrol. ${ }^{23}$

Angiogenesis involves a complicated pathological process including numerous types of inflammatory cells and cytokines. ${ }^{5,6}$ Macrophages play a key role in CNV, tumor angiogenesis, and other pathological angiogenic processes. ${ }^{7,8,10}$ Macrophages modulate angiogenesis by secreting various angiogenesis-related mediators, such as cyclooxygenase 2, IL-1 $\alpha$, IL-8, and MMPs. ${ }^{7,42-44}$ The authors' unpublished results suggest that macrophages could induce CNV by upregulating the expression of VEGF and MMP-9 in the cornea. In the present study, abundant macrophages were found in the sutured rat corneas, consistent with a previous study. ${ }^{11}$ Meanwhile, the control corneas expressed high levels of MCP-1 in mRNA and protein. MCP-1 is a potent macrophage-recruiting molecule. CNPs inhibited the expression of MCP-1 mRNA and protein and then suppressed macrophage infiltration in the cornea. The inhibition of macrophage and inflammatory cells infiltration showed the anti-inflammatory activity of celastrol in this model.

VEGF is a specific vascular endothelial cell mitogen and the central regulator of angiogenesis in various physiological and pathological conditions. ${ }^{45-48}$ In the present study, the expression of VEGF mRNA and protein were downregulated by the CNPs in the rat cornea. The role of VEGF in angiogenesis may explain the major reason celastrol inhibited $\mathrm{CNV}$ in this model. The downregulation of VEGF mRNA and protein in the cornea demonstrated the anti-angiogenic activities of CNPs. Degradation of the extracellular matrix is an important process in angiogenesis. MMPs are important factors that degrade the extracellular matrix. ${ }^{49,50}$ MMP-9, also known as gelatin B, plays a crucial role in degrading extracellular matrix and basement membrane during angiogenesis and organizational restructuring. ${ }^{51}$ 
Celastrol inhibits the migration and invasion of human breast carcinoma cells by downregulating MMP-9 expression in MCF-7 human breast cancer cells. ${ }^{52}$ In the present study, CNPs inhibited macrophage infiltration and accompanied the downregulation of MMP-9 at both the mRNA and protein levels in corneal tissue. This suggests that celastrol may block the development and progression of angiogenesis by altering extracellular matrix degradation. Celastrol can inhibit the production of TNF- $\alpha$, IL- 6 , and IL- $1 \beta$ in human monocytes, mouse macrophages, and microglial cells. ${ }^{16,53,54}$ However, results from the present study suggest that celastrol may suppress CNV by inhibiting macrophage infiltration and decreasing the expression of VEGF and MMP-9 in the cornea.

\section{Conclusion}

According to the authors' findings, two stitches can induce CNV in rats. This rat model is a highly stable, reproducible model and suitable for quantitative studies of CNV. PEG-b-PCL nanopolymeric micelles improve the water solubility of celastrol, a traditional Chinese herbal medicine. Celastrol release research shows a typical two-phase release profile containing a burst release followed by a sustained release.

Celastrol-loaded PEG-b-PCL nanopolymeric micelles inhibited the capillary structure formation and proliferation of HUVECs in a dose-dependent manner. CNPs suppress suture-induced CNV by inhibiting macrophage infiltration and reducing the expression of VEGF and MMP-9 at both the mRNA and protein level in the rat cornea. Celastrol shows potent anti-inflammatory and anti-angiogenic activities in this model. However, further studies are required to clarify the long-term side effects of celastrol on eyes and the signaling pathway utilized by celastrol to inhibit CNV.

\section{Acknowledgments}

This study was funded by the Natural Science Foundation of Guangdong Province (9451008901002232), the Doctoral Program Foundation of Institutions of Higher Education of China (20090171110084), and the Guangdong Provincial Department University-Industry Cooperation Projects (2010B090400415). The authors thank Professor Chuanbin $\mathrm{Wu}$ and Jinyuan Nie from the School of Pharmaceutical Sciences for their technical support in the high performance liquid chromatography analysis.

\section{Disclosure}

The authors report no conflicts of interest in this work.

\section{References}

1. Cursiefen C, Cao J, Chen L, et al. Inhibition of hemangiogenesis and lymphangiogenesis after normal-risk corneal transplantation by neutralizing VEGF promotes graft survival. Invest Ophthalmol Vis Sci. 2004;45(8):2666-2673.

2. Bauer D, Mrzyk S, van Rooijen N, Steuhl KP, Heiligenhaus A. Macrophage-depletion influences the course of murine HSV-1 keratitis. Curr Eye Res. 2000;20(1):45-53.

3. Stromblad S, Cheresh DA. Integrins, angiogenesis and vascular cell survival. Chem Biol. 1996;3(11):881-885.

4. Lee HS, Chung SK. The effect of subconjunctival suramin on corneal neovascularization in rabbits. Cornea. 2010;29(1):86-92.

5. Bernardini G, Ribatti D, Spinetti G, et al. Analysis of the role of chemokines in angiogenesis. J Immunol Methods. 2003;273(1-2):83-101.

6. Strieter RM, Burdick MD, Gomperts BN, Belperio JA, Keane MP. CXC chemokines in angiogenesis. Cytokine Growth Factor Rev. 2005;16(6):593-609.

7. David Dong ZM, Aplin AC, Nicosia RF. Regulation of angiogenesis by macrophages, dendritic cells, and circulating myelomonocytic cells. Curr Pharm Des. 2009;15(4):365-379.

8. Singh B, Lucci A. Role of cyclooxygenase-2 in breast cancer. $J$ Surg Res. 2002;108(1):173-179.

9. Szekanecz Z, Koch AE. Macrophages and their products in rheumatoid arthritis. Curr Opin Rheumatol. 2007;19(3):289-295.

10. Gabrusiewicz K, Ellert-Miklaszewska A, Lipko M, Sielska M, Frankowska M, Kaminska B. Characteristics of the alternative phenotype of microglia/macrophages and its modulation in experimental gliomas. PLoS One. 2011;6(8):e23902.

11. Usui T, Yamagami S, Kishimoto S, Seiich Y, Nakayama T, Amano S. Role of macrophage migration inhibitory factor in corneal neovascularization. Invest Ophthalmol Vis Sci. 2007;48(8):3545-3550.

12. Kannaiyan R, Shanmugam MK, Sethi G. Molecular targets of celastrol derived from Thunder of God Vine: potential role in the treatment of inflammatory disorders and cancer. Cancer Lett. 2011;303(1):9-20.

13. Huang L, Zhang Z, Zhang S, et al. Inhibitory action of celastrol on hypoxia-mediated angiogenesis and metastasis via the HIF-1 $\alpha$ pathway. Int J Mol Med. 2011;27(3):407-415.

14. Sethi G, Ahn KS, Pandey MK, Aggarwal BB. Celastrol, a novel triterpene, potentiates TNF-induced apoptosis and suppresses invasion of tumor cells by inhibiting NF-kappaB-regulated gene products and TAK1mediated NF-kappaB activation. Blood. 2007;109(7):2727-2735.

15. Huang Y, Zhou Y, Fan Y, Zhou D. Celastrol inhibits the growth of human glioma xenografts in nude mice through suppressing VEGFR expression. Cancer Lett. 2008;264(1):101-106.

16. Allison AC, Cacabelos R, Lombardi VR, Alvarez XA, Vigo C. Celastrol, a potent antioxidant and anti-inflammatory drug, as a possible treatment for Alzheimer's disease. Prog Neuropsychopharmacol Biol Psychiatry. 2001;25(7):1341-1357.

17. Huang FC, Chan WK, Moriarty KJ, et al. Novel cytokine release inhibitors. Part I: triterpenes. Bioorg Med Chem Lett. 1998;8(14):1883-1886.

18. Kim Y, Kim K, Lee H, et al. Celastrol binds to ERK and inhibits FcepsilonRI signaling to exert an anti-allergic effect. Eur J Pharmacol. 2009;612(1-3):131-142.

19. Jin HZ, Hwang BY, Kim HS, Lee JH, Kim YH, Lee JJ. Antiinflammatory constituents of Celastrus orbiculatus inhibit the NF-kappaB activation and NO production. $J$ Nat Prod. 2002;65(1):89-91.

20. Xiong MP, Yanez JA, Remsberg CM, et al. Formulation of a geldanamycin prodrug in mPEG-b-PCL micelles greatly enhances tolerability and pharmacokinetics in rats. J Control Release. 2008;129(1):33-40.

21. Mikhail AS, Allen C. Poly(ethylene glycol)-b-poly(epsilon-caprolactone) micelles containing chemically conjugated and physically entrapped docetaxel: synthesis, characterization, and the influence of the drug on micelle morphology. Biomacromolecules. 2010;11(5): 1273-1280.

22. Hseu YC, Chen SC, Lin WH, et al. Toona sinensis (leaf extracts) inhibit vascular endothelial growth factor (VEGF)-induced angiogenesis in vascular endothelial cells. J Ethnopharmacol. 2011;134(1):111-121. 
23. Pang X, Yi Z, Zhang J, et al. Celastrol suppresses angiogenesis-mediated tumor growth through inhibition of AKT/mammalian target of rapamycin pathway. Cancer Res. 2010;70(5):1951-1959.

24. Lv W, Chen L, Zhou DH, Wei B. Influence of specific blocking of the delta-like ligand 4/notch signal transduction pathway on the biological behavior of human umbilical vein endothelial cells. Cancer Biother Radiopharm. 2010;25(4):449-454.

25. Yoon SC, Kim JK, Kwak DH, et al. Antitumor activity of Soamsan, a traditional Korean medicine, via suppressing angiogenesis and growth factor transcription. J Ethnopharmacol. 2004;93(2-3):403-408.

26. Perez-Santonja JJ, Campos-Mollo E, Lledo-Riquelme M, Javaloy J, Alio JL. Inhibition of corneal neovascularization by topical bevacizumab (anti-VEGF) and sunitinib (anti-VEGF and anti-PDGF) in an animal model. Am J Ophthalmol. 2010;150(4):519-528. e1.

27. York M, Steiling W. A critical review of the assessment of eye irritation potential using the Draize rabbit eye test. $J$ Appl Toxicol. 1998;18(4):233-240.

28. $\mathrm{Su} \mathrm{W,} \mathrm{Li} \mathrm{Z,} \mathrm{Lin} \mathrm{M,} \mathrm{et} \mathrm{al.} \mathrm{The} \mathrm{effect} \mathrm{of} \mathrm{doxycycline} \mathrm{temperature-}$ sensitive hydrogel on inhibiting the corneal neovascularization induced by BFGF in rats. Graefes Arch Clin Exp Ophthalmol. 2011;249(3): 421-427.

29. Jia C, Zhu W, Ren S, Xi H, Li S, Wang Y. Comparison of genome-wide gene expression in suture- and alkali burn-induced murine corneal neovascularization. Mol Vis. 2011;17:2386-2399.

30. Seregin VV, Coffer JL. Biomineralization of calcium disilicide in porous polycaprolactone scaffolds. Biomaterials. 2006;27(27): 4745-4754.

31. Mondrinos MJ, Dembzynski R, Lu L, et al. Porogen-based solid freeform fabrication of polycaprolactone-calcium phosphate scaffolds for tissue engineering. Biomaterials. 2006;27(25):4399-4408.

32. Moon HT, Lee YK, Han JK, Byun Y. Improved blood compatibility by sustained release of heparin-deoxycholic acid conjugates in a PCL-PEG multiblock copolymer matrix. J Biomater Sci Polym Ed. 2002;13(7):817-828.

33. Huang MH, Li S, Hutmacher DW, et al. Degradation and cell culture studies on block copolymers prepared by ring opening polymerization of epsilon-caprolactone in the presence of poly(ethylene glycol). J Biomed Mater Res A. 2004;69(3):417-427.

34. Wei X, Gong C, Gou M, et al. Biodegradable poly(epsilon-caprolactone)poly(ethylene glycol) copolymers as drug delivery system. Int J Pharm. 2009;381(1):1-18.

35. Yanez JA, Forrest ML, Ohgami Y, Kwon GS, Davies NM. Pharmacometrics and delivery of novel nanoformulated PEGb-poly(epsilon-caprolactone) micelles of rapamycin. Cancer Chemother Pharmacol. 2008;61(1):133-144.

36. Forrest ML, Zhao A, Won CY, Malick AW, Kwon GS. Lipophilic prodrugs of Hsp90 inhibitor geldanamycin for nanoencapsulation in poly(ethylene glycol)-b-poly(epsilon-caprolactone) micelles. J Control Release. 2006;116(2):139-149.

37. Shuai X, Ai H, Nasongkla N, Kim S, Gao J. Micellar carriers based on block copolymers of poly(epsilon-caprolactone) and poly(ethylene glycol) for doxorubicin delivery. J Control Release. 2004;98(3):415-426.

38. Yin H, Gong C, Shi S, Liu X, Wei Y, Qian Z. Toxicity evaluation of biodegradable and thermosensitive PEG-PCL-PEG hydrogel as a potential in situ sustained ophthalmic drug delivery system. J Biomed Mater Res B Appl Biomater. 2010;92(1):129-137.
39. Samolov B, Steen B, Seregard S, van der Ploeg I, Montan P, Kvanta A. Delayed inflammation-associated corneal neovascularization in MMP-2-deficient mice. Exp Eye Res. 2005;80(2):159-166.

40. Jin Y, Arita M, Zhang Q, et al. Anti-angiogenesis effect of the novel anti-inflammatory and pro-resolving lipid mediators. Invest Ophthalmol Vis Sci. 2009;50(10):4743-4752.

41. Seo WY, Ju SM, Song HY, et al. Celastrol suppresses IFN-gammainduced ICAM-1 expression and subsequent monocyte adhesiveness via the induction of heme oxygenase- 1 in the HaCaT cells. Biochem Biophys Res Commun. 2010;398(1):140-145.

42. Koch AE, Polverini PJ, Kunkel SL, et al. Interleukin-8 as a macrophagederived mediator of angiogenesis. Science. 1992;258(5089):1798-1801.

43. Koga M, Kai H, Egami K, et al. Mutant MCP-1 therapy inhibits tumor angiogenesis and growth of malignant melanoma in mice. Biochem Biophys Res Commun. 2008;365(2):279-284.

44. Ho HH, Antoniv TT, Ji JD, Ivashkiv LB. Lipopolysaccharide-induced expression of matrix metalloproteinases in human monocytes is suppressed by IFN-gamma via superinduction of ATF-3 and suppression of AP-1. J Immunol. 2008;181(7):5089-5097.

45. Gille J, Swerlick RA, Caughman SW. Transforming growth factoralpha-induced transcriptional activation of the vascular permeability factor (VPF/VEGF) gene requires AP-2-dependent DNA binding and transactivation. EMBO J. 1997;16(4):750-759.

46. Malemud CJ. Growth hormone, VEGF and FGF: involvement in rheumatoid arthritis. Clin Chim Acta. 2007;375(1-2):10-19.

47. Wang S, Liu H, Ren L, Pan Y, Zhang Y. Inhibiting colorectal carcinoma growth and metastasis by blocking the expression of VEGF using RNA interference. Neoplasia. 2008;10(4):399-407.

48. Lohela M, Bry M, Tammela T, Alitalo K. VEGFs and receptors involved in angiogenesis versus lymphangiogenesis. Curr Opin Cell Biol. 2009;21(2):154-165.

49. Skotnicki JS, Zask A, Nelson FC, Albright JD, Levin JI. Design and synthetic considerations of matrix metalloproteinase inhibitors. Ann NY Acad Sci. 1999;878:61-72.

50. Thorns V, Walter GF, Thorns C. Expression of MMP-2, MMP-7, MMP-9, MMP-10 and MMP-11 in human astrocytic and oligodendroglial gliomas. Anticancer Res. 2003;23(5A):3937-3944.

51. Muroski ME, Roycik MD, Newcomer RG, et al. Matrix metalloproteinase-9/gelatinase B is a putative therapeutic target of chronic obstructive pulmonary disease and multiple sclerosis. Curr Pharm Biotechnol. 2008;9(1):34-46.

52. Kim Y, Kang H, Jang SW, Ko J. Celastrol inhibits breast cancer cell invasion via suppression of NF- $\kappa \mathrm{B}$-mediated matrix metalloproteinase-9 expression. Cell Physiol Biochem. 2011;28(2):175-184.

53. Kim DH, Shin EK, Kim YH, et al. Suppression of inflammatory responses by celastrol, a quinone methide triterpenoid isolated from Celastrus regelii. Eur J Clin Invest. 2009;39(9):819-827.

54. Jung HW, Chung YS, Kim YS, Park YK. Celastrol inhibits production of nitric oxide and proinflammatory cytokines through MAPK signal transduction and NF-kappaB in LPS-stimulated BV-2 microglial cells. Exp Mol Med. 2007;39(6):715-721.
International Journal of Nanomedicine

\section{Publish your work in this journal}

The International Journal of Nanomedicine is an international, peerreviewed journal focusing on the application of nanotechnology in diagnostics, therapeutics, and drug delivery systems throughout the biomedical field. This journal is indexed on PubMed Central,

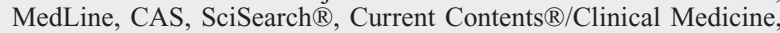

\section{Dovepress}

Journal Citation Reports/Science Edition, EMBase, Scopus and the Elsevier Bibliographic databases. The manuscript management system is completely online and includes a very quick and fair peer-review system, which is all easy to use. Visit http://www.dovepress.com/ testimonials.php to read real quotes from published authors. 